\title{
STUDYING THE SELF-ORGANIZATION OF MACROMOLECULES ON THE SURFACE OF TEXTURED POLYMER COATING OF METAL SHEET
}

\author{
${ }^{1}$ Olga MAKSIMOVA, ${ }^{2}$ Valentin SMIRNOV, ${ }^{2}$ Stepan OSIPOV, ${ }^{1}$ Evgenii MENSHIKOV, \\ ${ }^{2}$ Andrei MAKSIMOV, ${ }^{1}$ Vladislav EGOROV, ${ }^{2}$ Alena TRIFANOVA, ${ }^{1}$ Aleksander BAIDZHANOV, \\ ${ }^{2}$ Nadezhda OSIPOVA \\ ${ }^{1}$ Cherepovets State University, Laboratory of Mathematical and Computer Modeling, Cherepovets, \\ Russian Federation, og62@mail.ru \\ ${ }^{2}$ Cherepovets Stste University, Department of Physics, Cherepovets, Russian Federation
}

https://doi.org/10.37904/metal.2020.3566

\begin{abstract}
To describe a surface morphology of textured polymer coatings, the model of polymer chains attached to spherical granules was developed. By means of the model, we revealed the influence of coating density on its surface morphology. Diffuse reflection coefficient was calculated for polymer coatings with different morphologies.
\end{abstract}

Keywords: Textured polymer coating, associate, corrosion resistance, diffuse reflection coefficient

\section{INTRODUCTION}

The corrosion destruction is one of the main reasons for reduced efficiency of metal products $[1,2]$. One of the effective methods to prevent the corrosion is applying a polymer coating to steel sheets. The determination of corrosion resistance is an expensive procedure and requires a long test interval for its test (e.g., about 1 month in a salt spray chamber). The main indicator of the coating quality is the thickness of the polymer film. However, the production test data and the simulation results [3] show that there are manufacturing regimes which increase the polymer coating thickness for a given depth of the raw layer. It is economically profitable for a manufacturer to reduce the raw layer depth of polymer material and decrease artificially its density using special temperature conditions for the coating formation. Thus, the cost of production is reduced and it satisfies the quality requirements for polymer coating thickness [4]. However, an excessive decrease in the coating density leads to reduced corrosion resistance [5]. Therefore, the development of simple methods that allow determining the deviation of the polymer coating density from the standard one is a relevant task.

The objects of research in this paper are the surfaces of textured polymer coatings of the "Steel Velvet" type produced in PAO Severstal (Cherepovets, Russia). The texture of these coatings is created due to the presence of the filler consisting of polyacryl or polyamide granules in the paint material. During the drying process, polymer molecules are adsorbed on the particles surface, forming associates of the radial "viscous fingers" type [5], which are collected in star-shaped clusters scattered over the entire surface of the metal sheet (Figure 1). The surface morphology of such coatings depends on their formation regime and chemical structure of the paint material. If the temperature regime is violated, the sizes of associates change, they may be observed by means of an optical microscope. The aim of this work is to develop an optical method for detecting deviations of the polymer coating density from the standart one. 


\section{MODEL}

To study the sizes of associates, a model describing the arrangement of polymer chains near a spherical granule was developed. We relied on the fact that the associate near the granule has the shape of a rotation figure resembling a truncated cone. This is confirmed by the image obtained by the scanning probe microscopy method (Figure $1 \mathrm{c}$ ). The angle of the cone in the reference sample $\alpha=68^{\circ}$, the diameter of the associate $d=34.7 \mu \mathrm{m}$.

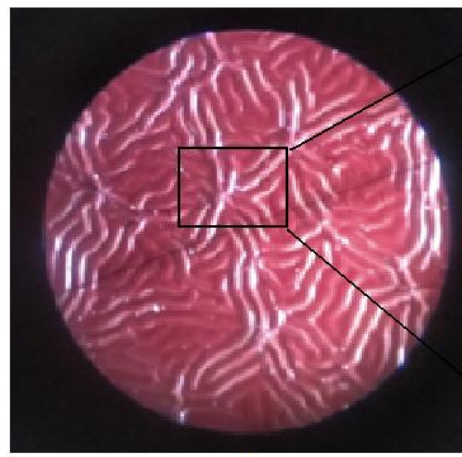

$a$

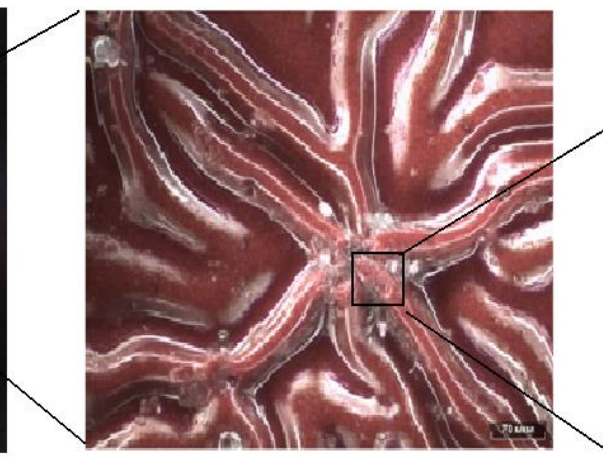

b

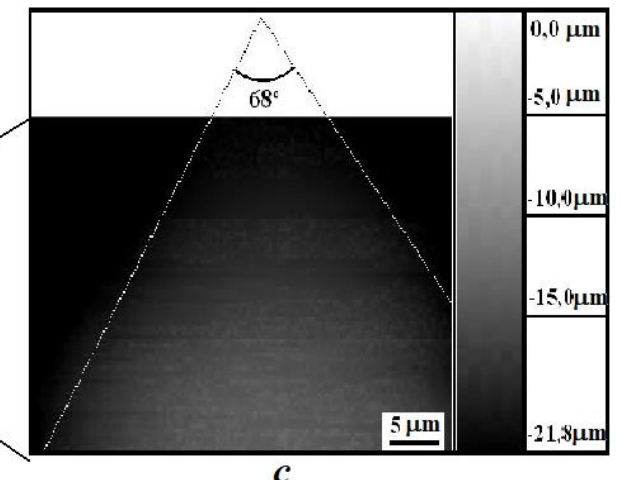

Figure 1 The surface image of rolled sheet metal with a polyester textured coating obtained by optical microscopes with different resolutions ( $a$ and $b$ ) and scanning probe microscopy (c)

In the proposed model, it is assumed that polymer chains are adsorbed on a spherical surface of the radius $R$ (Figure 2), forming supramolecular structures (associates). The angular size of such structure is limited by the solid angle $\alpha$ (Figure 2 a). The position of an individual polymer chain inside the finger is characterized by the angle $\theta$ (Figure $\mathbf{2} \mathbf{b}$ ). One chain ends are adsorbed on the spherical granule surface, the opposite ends are stretched. Each chain can be divided into three sections $A B, B C$ and $C D$ with lengths $l_{1}, l_{2}$ and $l_{3}$ respectively. The intermolecular distances in the section $A B$ are equal to $b_{0}$ and they are determined by the chemical structure of the polymer, the intermolecular distances near the granule (in the section CD) $b_{g}$ depend also on the chemical composition of the granule. Therefore, the values $b_{0} и b_{g}$ are different. The ratio between these distances is defined by the ratio $g=b_{0} / b_{g}$.

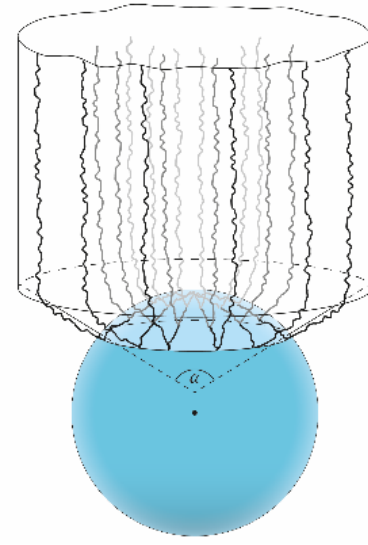

a

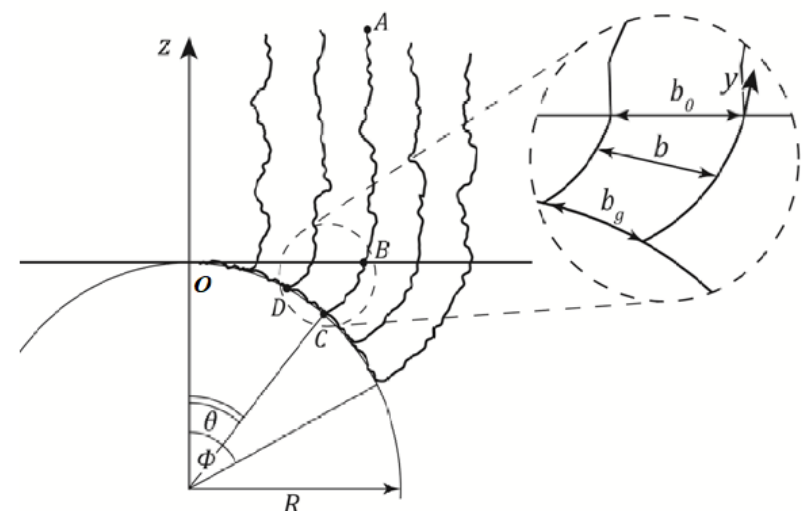

b

Figure 2 Model of the polymer system near the granule

Section CD. The potential energy is calculated by the formula

$w_{1}=-\psi \cdot l_{3}$, 
where the factor $\psi$ characterizes the adsorption strength as the corresponding parameter in the ref. [7].

Section BC. It is assumed that the interchain distance $b$ in this section varies linearly along the $y$-axis from $b_{g}$ (at the point $C$ ) to $b_{0}$ (at the point $B$ ). The potential energy in this section consists of two terms.

Lennard-Jones Potential for two chain links with the length link, located in adjacent chains is defined as

$$
w_{2 L D}=\frac{4}{l_{\text {link }}} \int_{0}^{l_{2}(\theta)} \varepsilon\left(\frac{b_{0}^{12}}{b^{12}(y)}-\frac{2 \cdot b_{0}^{6}}{b^{6}(y)}\right) d y,
$$

where $\varepsilon$ is the depth of the potential well. We took into account interactions with only four nearest neighbors, since Lennard-Jones potential decreases greatly with distance.

The energy of orientational interactions. This term was calculated in the framework of the Hurst and Harris [8] model, in the mean field approximation developed in the Maier -Saupe theory [9]. To simplify the description of the effects related with intermolecular orientational interactions, we introduce the mean (molecular) field $\mu \cdot V$, where the quantity $\mu$ is the long-range orientational order parameter for kinetic chain units. The mean field $V$ is created by dipole-dipole interactions between chain units and its value is inversely proportional to the value of the interchain distance. Therefore, $V \sim \sqrt{\rho}$, where the quantity $\rho$ is the density of the polymer film. The energy of orientation interactions is calculated by the formula

$$
w_{2 o r}(\theta)=\int_{0}^{l_{2}(\theta)} \mu_{2}^{2}(\theta) \frac{V \cdot b_{0}}{b(y)} d y,
$$

where the long range orientation order parameter $\mu_{2}(\mathrm{y})$ is determined from the solution of the Langevin equation

$\mu_{2}(y)=L(x) \cdot\left(\frac{\mu_{2}(y) \cdot V(y)}{k_{B} \cdot T}\right)$,

where $L(\mathrm{x})$ is the Langevin function, $T$ is the peak temperature of coating formation $(\mathrm{K})$.

Section $\mathrm{AB}$. The potential energy also consists of two terms. It is assumed that the intermolecular distance $b_{0}$ in this section is constant. Lennard-Jones Potential in this section is given by

$$
w_{1 L D}=-\frac{4 \cdot \varepsilon \cdot l_{1}}{l_{\text {link }}} .
$$

The potential of orientation interactions is calculated by the following expression

$$
w_{1 o r}=-\mu_{1}^{2}(y) \cdot V \text {, }
$$

where the value of the long-range orientation parameter $\mu_{1}(\mathrm{y})$ is also determined from the solution of the Langevin equation (4).

The length of the arc $\mathrm{OC}$ is equal to $R \cdot \theta$, and the length of the segment $\mathrm{OB}$ is equal to $R \cdot \theta \cdot g$. Therefore, for a given angle $\Theta$ the length of the section $k_{2}$ is calculated by the formula

$$
l_{2}(\theta)=R \cdot \sqrt{(g \cdot \theta-\sin (\theta))^{2}+(1-\cos (\theta))^{2}} .
$$

The average ratio between lengths $l_{1}$ and $l_{3}$ is determined from the Boltzmann distribution 
$\frac{l_{1}}{L_{\text {chain }}-l_{2}}=\frac{\int_{0}^{L_{\text {chain }}-l_{2}} z \cdot \exp \left(-\frac{w_{1}+w_{3}}{k_{B} \cdot T}\right) d z}{\int_{0}^{L_{\text {chain }}-l_{2}} \exp \left(-\frac{w_{1}+w_{3}}{k_{B} \cdot T}\right) d z}, \quad l_{3}=L_{\text {chain }}-l_{2}-l_{1}$,

where $L_{\text {chain }}$ is the contour length of the chain.

The total energy of one chain is equal to

$w(\theta)=w_{1}(\theta)+w_{2 o r}(\theta)+w_{2 L D}(\theta)+w_{3}(\theta)$.

The energy of the polymer chains located inside the associate (Figure $\mathbf{2}$ a) is equal to

$W(\Phi)=2 \cdot \pi \cdot R^{2} \cdot c \cdot \int_{0}^{\Phi} w(\theta) \cdot \sin (\theta) d \theta$,

where the quantity $c$ is the concentration of macromolecules (the number of chain links adsorbed per unit area of the granule).

\section{DETERMINATION OF MODELING PARAMETERS}

To determine the simulation parameters, it is assumed that the associates are arranged on the granule as a dense package of spherical caps on the sphere. The density of such package is approximately equal to 0.9069 . The area of one associate on a granule is equal to $2 \cdot \pi \cdot R^{2} \cdot(1-\cos \Phi)$ or $0.9069 \cdot\left(4 \cdot \pi \cdot R^{2}\right) / N$, where $N$ is number of associates. Therefore, the angle $\Phi$ can be calculated using the formula

$\Phi=\arccos \left(1-\frac{1.81}{N}\right)$

In the image of the reference sample (Figure $\mathbf{1} \mathbf{b}$ ), we can observe only the upper half of the granule, on the surface of which 6 associates are located. Therefore, the total number associates $N=12$. Calculations have shown that in the reference sample, the angle $\Phi=33^{\circ}$. Using the scanning probe microscopy method (Figure $1 \mathrm{c}$ ), we have determined the value of the angle $\alpha=68^{\circ}$. The formula for the ratio between the angles $\Phi$ and $\alpha$ has been obtained.

$\cos \left(\frac{\alpha}{2}\right)=\frac{1-\cos \Phi}{\sqrt{(g \cdot \Phi-\sin \Phi)^{2}+(1-\cos \Phi)^{2}}}$.

Calculations have shown that for the considered system, the value of $g=1.14$.

Figure 3 shows the dependence of the potential energy of one associate vs. the angle $\Phi$. The simulation parameters have been selected from the condition that the potential energy has a minimum at the angle $\Phi=33^{\circ}$ (orange curve)

\section{RESULTS OF THE SIMULATION}

For the chosen parameters, the angle $\Phi$ changes insignificantly with varying the temperature. The simulation results for other parameters have shown that the width of the associate decreases with increase in the final temperature (the peak temperature of the metal). However, the structure of the polymer system is determined not only by the final temperature, but also by the time during which the system was at high temperatures. In this case, according to the Arrhenius equation, the length of the polymer chain increases. Figure 3 shows that 
increase in the chain length leads to decrease in the angle $\Phi$, and, consequently, to decrease in the associate width $d$ and increase in the number of rays in the star-shaped cluster (Figure 1 a). Calculations have shown that the thickness $d$ does not depend on the granule size. Therefore, for modeling the surface morphology of a textured polymer coating on a solid, the granule size may be chosen arbitrarily.

Figure 4 shows the angle $\Phi$ vs. the density of the polymer film. As can be seen from the figure, the angle $\Phi$ decreases with the decrease in the coating density. This leads to a decrease in the width of the associate and an increase in the number of rays in the star-shaped cluster (Figure $1 \mathbf{a}$ ). Therefore, a violation of the temperature regime of coating formation may be determined by comparing the surface texture of the product and the standard by means of optical microscope. The sharp increase in the angle $\Phi$ is explained by the existence of a structural transition in the polymer system.

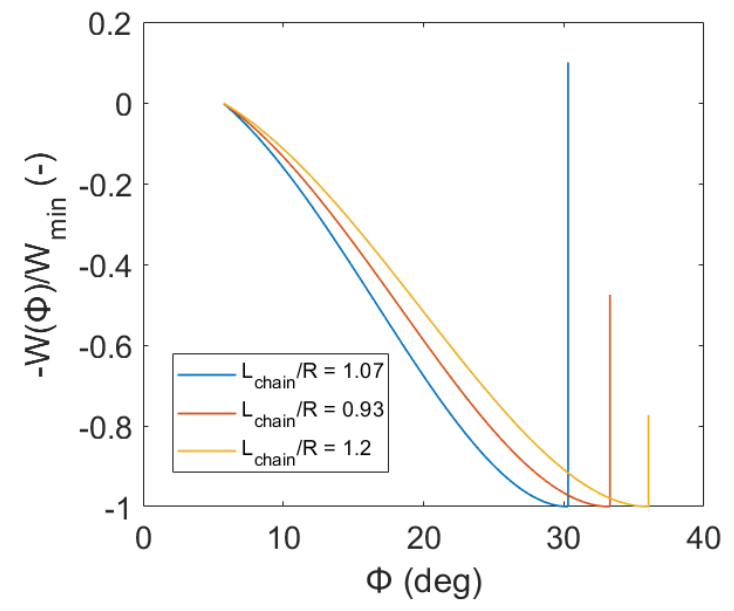

Figure 3 The potential energy of the associate vs. the angle $\Phi . V / \varepsilon=60, \psi / \varepsilon=1, k_{B} \cdot T / \varepsilon=20$

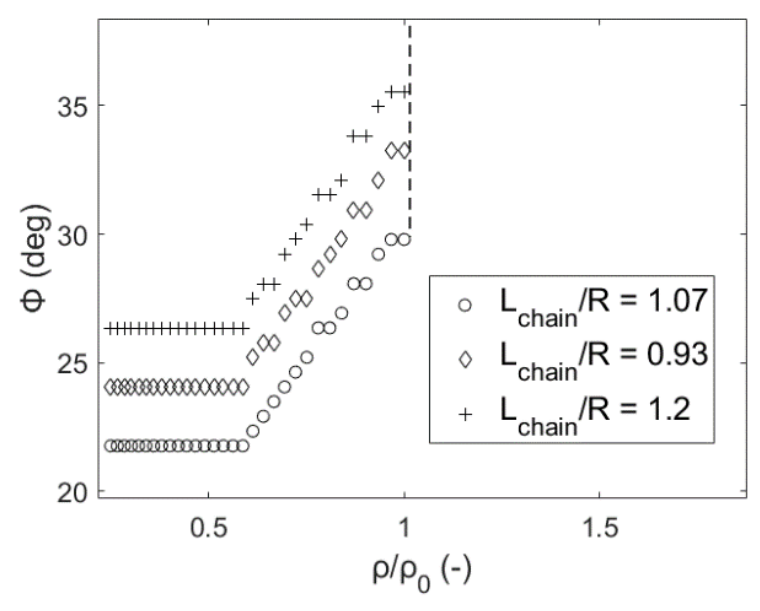

Figure 4 The angle $\Phi$ vs. the coating density $\rho$ of the reference sample

\section{DIFFUSE REFLECTION COEFFICIENT}

The optical properties of the coating are affected by its surface morphology. Therefore, the influence of the associate's size on the value of the diffuse reflection coefficient (DRC) was investigated. The formulas given in ref. [10] were used for a quasiperiodic surface when it is illuminated normally to the metal surface.

$$
R_{d r}=2 \cdot \pi \cdot \int_{0}^{\pi / 4} f(\beta) \cdot \sin \beta \cdot \cos \beta \cdot r_{F}(\beta) d \beta,
$$

where $\gamma_{\mathrm{m}}$ is the maximum value of the tangent of the angle of sine wave inclination to the metal plane,

$$
f(\beta)=\frac{4 \cdot \arccos \left[\tan \beta / \gamma_{m}\right]}{\pi^{3} \cdot \sin \beta \cdot \cos ^{3} \beta \cdot \sqrt{\gamma_{m}^{2}-\tan ^{2} \beta}},
$$

The Fresnel coefficient $r_{\mathrm{F}}(\beta)$ in Eq. (13) at normal incidence of natural light is determined by the formula

$$
r_{F}(\beta)=\frac{1}{2}\left(\frac{\tan ^{2}\left(\beta-\arcsin \left(\frac{n_{1}}{n_{2}} \cdot \sin \beta\right)\right)}{\tan ^{2}\left(\beta+\arcsin \left(\frac{n_{1}}{n_{2}} \cdot \sin \beta\right)\right)}+\frac{\sin ^{2}\left(\beta-\arcsin \left(\frac{n_{1}}{n_{2}} \cdot \sin \beta\right)\right)}{\sin ^{2}\left(\beta+\arcsin \left(\frac{n_{1}}{n_{2}} \cdot \sin \beta\right)\right)}\right),
$$


where the quantities $n_{1}$ and $n_{2}$ are the refractive indices of light in air and in the polymer, respectively. Figure 5 shows the DRC vs. the width of the associate for different values of its height $h$. As can be seen from

the figure, the DRC increases almost linearly with an increase in the associate width. Meanwhile, an increase in its height leads to a decrease in the DRC.

\section{CONCLUSION}

The simulation results have shown that the surface morphology of the textured polymer coating on the metal sheet is determined not only by the chemical structure of the polymer material, but it is also sensitive to changes in the temperature regime of its formation. For example, artificial reducing the density of the coating leads to a decrease in the size of the associate and a decrease in the DRC.

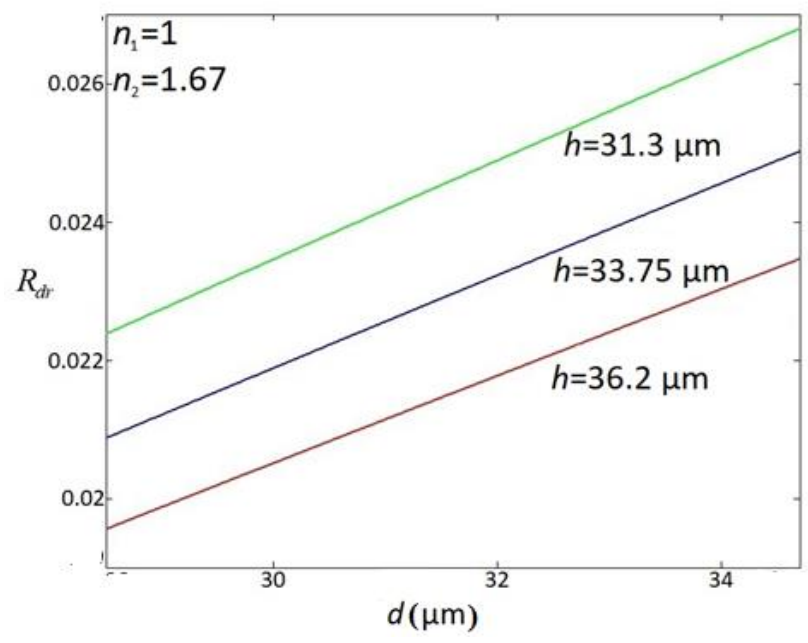

Figure $5 \mathrm{DRC}$ vs. the width of the associate at different values of its height

\section{ACKNOWLEDGEMENTS}

This study was supported by the Russian Foundation for Basic Research, project No. 19-42-350001.

\section{REFERENCES}

[1] McCAFFERTY, Edward. Introduction to Corrosion Science. New York: Springer, 2009.

[2] MENDALA, J., KOZUBA, J. The influence of sodium nitrite concentration in inhibitor protection of structural elements working in aggressive environmental conditions. In METAL 2019: 28th International Conference on Metallurgy and Materials. Ostrava: Tanger, 2019, pp. 1097-1102.

[3] MAKSIMOVA, O.G., MAKSIMOV, A.V., MOISEEVA, A.I. Influence of interface on the formation process of polymer coatings on metal. Journal of Advanced Dielectrics. 2016, vol. 6, no. 1, p. 1650004.

[4] GOST 34180-2017 Steel cold rolled and cold rolled hot-galvanized sheet with polymer coating, prepainted by the continuous coil-coating process. Specifications. Moscow: Standartinform, 2017.

[5] MAKSIMOVA, O.G., MAKSIMOV, A.V., BAIDGANOV, A.R. Simulation of conductivity of polymer films on metal surface. Journal of Physics: Conference Series. 2015, vol. 633, p. 012049.

[6] NITTMAN, J., DACCORD, G., STANLEY, H.E. In Proceedings of the Sixth Trieste International Symposium on Fractals in Physics. Trieste: ICTP, 1985.

[7] KLUSHIN, L.I., SKVORTSOV, A.M., GORBUNOV, A.A. Adsorption of a macromolecule in an external field: An exactly solvable model with bicritical behavior. Physical Review E. 1997, vol. 56, pp. 1511-1521.

[8] HARRIS, R.A., HEARST, J. E. Journal of Chemical Physics. 1978, vol. 44, p. 2595.

[9] MAIER, W., SAUPE, A. Eine einfache molekulare Theorie des nematischen kristallinflüssigen Zustandes. Zeitschrift fur Naturforschung A. 1958, vol. 13, no. 3, pp. 564-570.

[10] MAIER, W., SAUPE, A. Eine einfache molecular-statistische Theorie der nematischen kristallinflüssigen Phase.Teil I. Zeitschrift fur Naturforschung A. 1959, vol. 14, no. 10, pp. 882-889.

[11] MAIER, W., SAUPE, A. Eine einfache molecular-statistische Theorie der nematischen kristallinflüssigen Phase.Teil II. Zeitschrift fur Naturforschung A. 1960, vol. 15, no. 7, pp. 287-292.

[12] BARUN, V.V., IVANOV, A.P. Light scattering by a rough surface of human skin. 2. Diffuse reflectance. Quantum Electronics. 2013, vol. 43, no. 10, pp. 979-987. 